\title{
Leveraging biological and statistical covariates improves the detection power in epigenome-wide association testing
}

Jinyan Huang ${ }^{1{ }^{*}}$, Ling Bai ${ }^{1 \dagger}$, Bowen Cui ${ }^{1 \dagger}$, Liang Wu' ${ }^{1}$ Liwen Wang ${ }^{2}$, Zhiyin An ${ }^{1}$, Shulin Ruan ${ }^{1}$, Yue $\mathrm{Yu}^{3}$, Xianyang Zhang ${ }^{4^{*}}$ and Jun Chen ${ }^{5^{*}}$

\begin{abstract}
Background: Epigenome-wide association studies (EWAS), which seek the association between epigenetic marks and an outcome or exposure, involve multiple hypothesis testing. False discovery rate (FDR) control has been widely used for multiple testing correction. However, traditional FDR control methods do not use auxiliary covariates, and they could be less powerful if the covariates could inform the likelihood of the null hypothesis. Recently, many covariate-adaptive FDR control methods have been developed, but application of these methods to EWAS data has not yet been explored. It is not clear whether these methods can significantly improve detection power, and if so, which covariates are more relevant for EWAS data.

Results: In this study, we evaluate the performance of five covariate-adaptive FDR control methods with EWASrelated covariates using simulated as well as real EWAS datasets. We develop an omnibus test to assess the informativeness of the covariates. We find that statistical covariates are generally more informative than biological covariates, and the covariates of methylation mean and variance are almost universally informative. In contrast, the informativeness of biological covariates depends on specific datasets. We show that the independent hypothesis weighting (IHW) and covariate adaptive multiple testing (CAMT) method are overall more powerful, especially for sparse signals, and could improve the detection power by a median of $25 \%$ and $68 \%$ on real datasets, compared to the ST procedure. We further validate the findings in various biological contexts.
\end{abstract}

Conclusions: Covariate-adaptive FDR control methods with informative covariates can significantly increase the detection power for EWAS. For sparse signals, IHW and CAMT are recommended.

Keywords: False discovery rate, EWAS, Multiple hypothesis testing, Covariate

\footnotetext{
*Correspondence: huangjy@sjtu.edu.cn; zhangxiany@stat.tamu.edu;

chen.jun2@mayo.edu

${ }^{\dagger}$ Jinyan Huang, Ling Bai and Bowen Cui contributed equally to this work.

'State Key Laboratory of Medical Genomics, Shanghai Institute of

Hematology, National Research Center for Translational Medicine, Rui-Jin

Hospital, Shanghai Jiao Tong University School of Medicine, Shanghai Jiao

Tong University, 197 Ruijin Er Road, Shanghai 200025, China

${ }^{4}$ Department of Statistics, Texas A\&M University, Blocker 449D, College

Station, TX 77843, USA

${ }^{5}$ Division of Biomedical Statistics and Informatics, Department of Health

Sciences Research and Center for Individualized Medicine, Mayo Clinic, 200

1st St SW, Rochester, MN 55905, USA

Full list of author information is available at the end of the article
}

\section{$\triangle B M C$}

C C The Author(s). 2020 Open Access This article is licensed under a Creative Commons Attribution 4.0 International License, which permits use, sharing, adaptation, distribution and reproduction in any medium or format, as long as you give appropriate credit to the original author(s) and the source, provide a link to the Creative Commons licence, and indicate if changes were made. The images or other third party material in this article are included in the article's Creative Commons licence, unless indicated otherwise in a credit line to the material. If material is not included in the article's Creative Commons licence and your intended use is not permitted by statutory regulation or exceeds the permitted use, you will need to obtain permission directly from the copyright holder. To view a copy of this licence, visit http://creativecommons.org/licenses/by/4.0/ The Creative Commons Public Domain Dedication waiver (http://creativecommons.org/publicdomain/zero/1.0/) applies to the data made available in this article, unless otherwise stated in a credit line to the data. 


\section{Background}

DNA methylation, as a major form of epigenetic modifications, plays a vital role in various biological processes including cell differentiation [1-3], genomic imprinting [4], gene transcription, and X-chromosome inactivation [5]. The landscape of DNA methylation is not only associated with normal physiological phenomena such as aging [6-8], but also with many human diseases including cancers [8-10], atherosclerosis [11], and Alzheimer's disease [12, 13]. While the DNA sequences are relatively constant throughout life, DNA methylation is dynamic and modifiable, providing promising therapeutic targets for disease treatment [14-16].

With the advance of high-throughput genomic technologies, DNA methylation can now be interrogated at the genome scale. Although the gold standard for DNA methylation measurement remains bisulfite sequencing [17], DNA methylation arrays, due to its low cost, high reproducibility, and good genome coverage, have been widely used in genome-wide methylation analyses. Illumina's Infinium Human Methylation 450K BeadChip and EPIC BeadChip, which cover more than 450,000 and 850,000 CpG methylation sites respectively, are two predominant products in the market. The availability of these high-density methylation arrays has fueled epigenome-wide association studies (EWAS), which seek to identify methylation variants associated with an outcome or exposure of interest [18-23]. Analysis of EWAS data typically involves testing all the CpG sites simultaneously, leading to a massive multiple testing problem. Two statistical approaches have been developed to address multiple testing: family-wise error rate (FWER) and false discovery rate (FDR) control. The FWER approach controls the probability of making one or more false discoveries, while the FDR approach controls the expected proportion of false discoveries. Therefore, the FWER approach, such as Bonferroni correction [24], offers a more stringent type I error control but is substantially less powerful than the FDR approach. For EWAS data, the sample size and the expected effect size are usually moderate, making the FDR approach particularly appealing [25-27]. Among existing FDR control procedures, the original Benjamini-Hochberg step-up procedure (BH) [28] and Storey's $q$ value procedure (ST) [29] are the two most popular methods for genome-wide multiple testing. Compared to the $\mathrm{BH}$ procedure, the ST procedure considers the proportion of null hypotheses and is more powerful when the signal is dense.

Both $\mathrm{BH}$ and ST procedures do not differentiate hypotheses, that is, they assume that each hypothesis is equally likely to be true or false, and their rejection rule is based solely on the $p$ values. Therefore, $\mathrm{BH}$ and ST procedures may not be optimal when we have additional information about the hypotheses in terms of their null probability (the probability for the null hypothesis to be true) or statistical power. For EWAS data, besides the association $p$ values, we have plenty of auxiliary covariates, which could be informative of the null probability or statistical power of the CpG-specific hypotheses. For example, differentially methylated CpG positions (DMPs) have been found to be enriched in specific genomic regions, such as promoters [30, 31], CpG islands [20], shores [32], a specific chromosome [33], and DNase I hypersensitive sites [32, 34]. In addition, some studies found that DMPs tend to change in the same direction, especially in cancer, where genomewide hypomethylation or hypermethylation has been frequently observed [10]. Other studies found a large portion of the CpGs are subject to large measurement errors, hence low statistical power $[35,36]$. Therefore, incorporating such biological or statistical covariates in FDR control could potentially improve the power to detect DMPs.

Recently, there has been a surge in the development of statistical methodologies for FDR control procedures accommodating covariates with the aim to improve the detection power while still maintaining the target FDR level. Such covariate-adaptive FDR control methods include weighted FDR [37], the conditional local FDR (LFDR) [38], FDR regression (FDRreg) [39], independent hypothesis weighting (IHW) [40], adaptive shrinkage (ASH) [41], Boca and Leek's FDR regression (BL) [42], adaptive $p$ value thresholding (AdaPT) [43], and covariate adaptive multiple testing (CAMT) [44]. Although these methods differ in their respective model and input, they share the same idea: by relaxing the rejection criterion for more promising hypotheses based on the covariate information and tightening the criterion for others, substantial power improvement can be achieved without affecting the target FDR level. These covariate-adaptive methods have been demonstrated to be superior to traditional $\mathrm{BH} / \mathrm{ST}$ procedures by case studies on ChIP-seq, genotype, microbiome, RNA-seq, and scRNA-seq data [45], but application to EWAS data has not yet been attempted. Since these methods rely on the assumption that the hypotheses are independent or weakly dependent, it is not clear whether they are robust to the typical correlation structure observed in EWAS data. Moreover, it is unknown what CpG-related covariates are relevant. Therefore, a rigorous and comprehensive evaluation of the covariate-adaptive FDR methods for EWAS data is critical before recommending them to the field.

In this study, we compared five covariate-adaptive FDR control methods using real data-based simulations and investigated the performance of $14 \mathrm{CpG}$-related covariates on 61 EWAS datasets. The contribution of the paper is thus threefold: (1) we developed a powerful statistical test for detecting and selecting informative covariates, (2) we identified the most robust and powerful covariate-adaptive FDR control methods for EWAS data, and (3) we recognized the most relevant covariates for EWAS data. 


\section{Results}

Overview of the EWAS datasets and covariates selected for evaluation

In this study, 61 EWAS datasets were collected based on 58 Gene Expression Omnibus (GEO) methylation datasets whose platforms were Infinium Human Methylation 450K BeadChip. The sample size of EWAS datasets ranged from 100 to 689 , with an average of 211. Details about the data source and analyzed phenotypes can be found in the additional file (Additional file 1: Table S1). The methylation datasets came from diverse tissue sources, including blood, brain, lung, breast, colorectal, liver, esophagus, and others (Additional file 6: Figure S1). Around half of the datasets were from the blood (30/61). After quality control, we performed surrogate variable analysis (SmartSVA) [46] to capture significant sources of methylation variability, such as cellular heterogeneity [47] and batch effects [48]. The constructed surrogate variables were included as covariates in the regression model to account for potential confounding effects. After adjusting for surrogate variables, we observed a significant reduction of the genomic inflation [46] of the association $p$ values (Additional file 6: Figure S2). The adjusted $p$ values were then to be corrected for multiple testing by various FDR control procedures.

Covariate-adaptive FDR control methods require selection of appropriate covariate(s) for the method to work. To be statistically valid, the covariate should be independent of the $p$ values under the null. Meanwhile, in order to increase the detection power, the covariate should be informative of the prior null probability or statistical power of the underlying hypotheses. For EWAS data, we investigated 14 potential covariates, which were assumed to possess the aforementioned properties. These covariates can be classified as statistical covariates (internal) and biological/technical covariates (external). Statistical covariates are related to the statistical properties of the methylation data. We investigated mean of the Beta-value ("mean"), standard deviation of the Beta-value ("sd.b") or M-value ("sd.m"), median absolute deviation of the Beta-value ("mad", a robust measure of variance), measure of unimodality of the Beta-value ("dip"), inverse precision parameter of the Beta-value ("precision") [49], sign of the regression coefficients ("direction") for binary/continuous phenotype, and intraclass correlation coefficient (ICC) using the Beta-value ("icc.b"), or M-value ("icc.m") when replicates were available. Biological/technical covariates describe the biological/technical properties of the CpGs and are based on the annotation of the CpG probes. We considered the location in the gene region ("refgene.pos"), relation to the CpG island ("cpg.loc"), chromosome number ("chr"), DNase I hypersensitive site ("dhs"), and Infinium probe type ("probe.type"). Details of the covariates are listed in Table 1.

\section{An omnibus test to assess the informativeness of the covariate for multiple testing adjustment}

For a covariate that could be leveraged to increase the power for epigenome-wide multiple testing, it has to be informative of the null probability or the statistical

Table 1 Description of the covariates evaluated

\begin{tabular}{|c|c|c|}
\hline Covariate & Definition & Type \\
\hline mean & Mean Beta-values (R base::mean) & Statistical, continuous \\
\hline sd.b/sd.m $m^{\# 1}$ & Standard deviation of Beta-value or M-value (R stats.:.sd) & Statistical, continuous \\
\hline mad & Median absolute deviation ( $R$ stats:::mad) & Statistical, continuous \\
\hline dip & Measure of unimodality using dip statistic (R diptest::dip) & Statistical, continuous \\
\hline precision & Inverse precision parameter, $\left(1 /\left(\right.\right.$ mean $\times(1-$ mean $\left.\left.) / s d^{2}-1\right)\right)$ & Statistical, continuous \\
\hline direction $^{\# 2}$ & Sign of regression coefficients & $\begin{array}{l}\text { Statistical, categorical } \\
\{\text { positive, negative\} }\end{array}$ \\
\hline icc.b/icc.m $\mathrm{m}^{\# 3}$ & Intraclass correlation coefficient (R CpGFilter::CpGFilterICC) & Statistical, continuous \\
\hline refgene.pos & Position in the gene region & $\begin{array}{l}\text { Biological, categorical } \\
\left\{5^{\prime} U T R, T S S 1500, T S S 200,1 \text { stExon, Body, 3'-UTR, Non_gene } e^{\# 4}\right\}\end{array}$ \\
\hline cpg.loc & Relation to CpG island & $\begin{array}{l}\text { Biological, categorical } \\
\text { \{OpenSea, N_Shelf, N_Shore, Island, S_Shore, S_Shelf\} }\end{array}$ \\
\hline chr & Chromosome number & $\begin{array}{l}\text { Biological, categorical } \\
\{c h r 1, \text { chr } 2, \ldots, c h r 22\}\end{array}$ \\
\hline dhs & DNase I hypersensitive site & $\begin{array}{l}\text { Biological, categorical } \\
\text { \{yes, no\} }\end{array}$ \\
\hline probe.type & Infinium probe type & $\begin{array}{l}\text { Technical, categorical } \\
\text { \{type I, type II\} }\end{array}$ \\
\hline
\end{tabular}

\#1. The suffix .b and .m stand for Beta-value and M-value, respectively. If not noted, the calculation is based on Beta-value

\#2. Used when the phenotype is binary or continuous

\#3. Used when there are technical replicates in data

\#4. When multiple gene regions are annotated for the CpG, the first one is used. If no annotation is available, we labeled it "Non_gene" 
power, either of which will lead to the dependency between the $p$ value and the covariate. Such dependency is usually explored by various diagnostic plots such as scatter plot and stratified $p$ value histogram [40] (Additional file 6: Figure S3 \& 4). Although these diagnostic plots can detect a strong dependency efficiently, they may have limited ability to reveal a more subtle and complex relationship, especially when the signal is sparse. We thus develop a formal statistical test to rigorously assess the dependency between $p$ value and the covariate ("Methods"). Basically, the test exploits the assumptions that the signal is usually sparse, and the dependency may be highly nonlinear. This is achieved by testing the association between two categorical variables after dichotomizing the $p$ values at the lower end and splitting the covariate into disjoint sets if it is continuous. An omnibus-type test is designed to combine evidence through various categorizations, and permutation is used to assess the statistical significance.

We first performed simulations to assess the type I error and power of the proposed omnibus test and benchmarked it against the naïve tests-Spearman's rank test and Kruskal-Wallis test for continuous and categorical covariates, respectively. We generated data with both continuous and categorical covariates and varied the degree of $p$ value-covariate dependency and signal density ("Methods"). For a continuous covariate, we investigated both monotonic and non-monotonic dependency. The results are summarized in Fig. 1a. When there was no dependency (the covariate was not informative), both the omnibus and naïve tests controlled the type I error at the nominal level under all settings. When the signal was sparse, the omnibus test was significantly more powerful than the naïve tests. As the signal became denser, the power difference decreased for categorical covariates and continuous covariates under a monotonic relationship. However, when the relationship was non-monotonic, the naïve tests were powerless under all settings. Therefore, the proposed omnibus test was particularly suited for the sparse signal and complex dependency setting, which were expected in many EWAS datasets.

\section{Evaluation of the informativeness of EWAS-relevant covariates}

We next applied the omnibus test to the aforementioned 14 covariates based on the CpG association $p$ values from the 61 EWAS datasets. To satisfy the exchangeability assumption of the permutation-based omnibus test, we subsampled the $p$ values so that the auto-correlation coefficient was close to 0 between adjacent CpGs. We plotted the distribution of the omnibus test $p$ values (log scale) for these 14 covariates to assess their informativeness (Fig. 1b, Additional file 2: Table S2). One clear pattern is that the statistical covariates dominate the biological/technical covariates with overall smaller omnibus $p$ values. Interestingly, among the statistical covariates, the mean methylation level ("mean") was the most

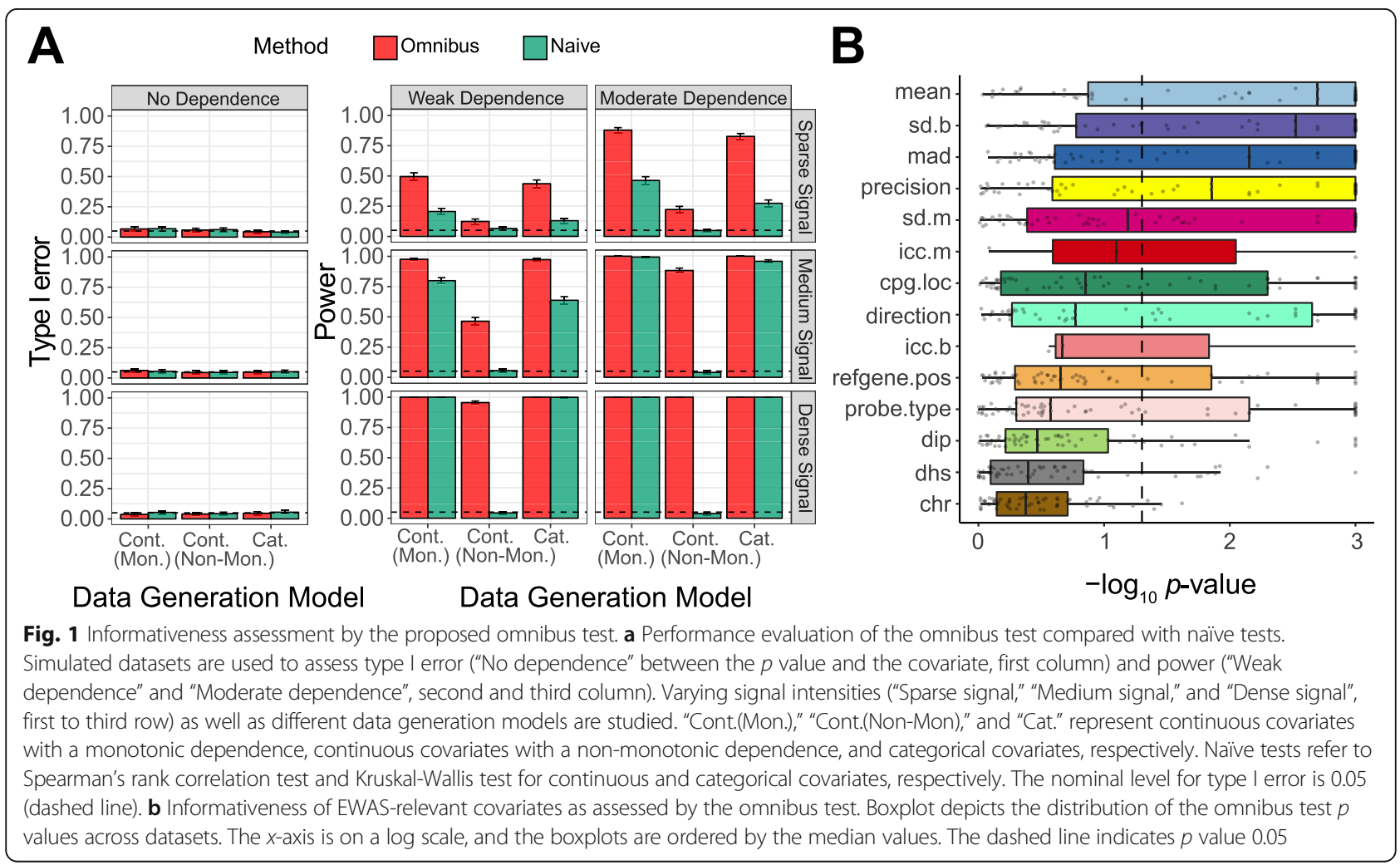


informative, followed by the covariates measuring the methylation variance ("sd.b," "mad," "precision"). For these covariates, the majority of datasets achieved significant omnibus test $p$ values $(p<0.05)$. Additional file 6 : Figure S3A\&B shows two examples where the small association $p$ values were enriched in small and medium Beta-values, respectively. Variance on the methylation Beta-value ("sd.b") was more informative than that on the M-value ("sd.m"). The direction of the effect ("direction") had mixed results, with $46.3 \%$ (25/54) of the datasets being significant. The DIP statistic ("dip"), on the other hand, was the least informative; only 12 out of 61 datasets had significant $p$ values. For the three datasets with replicates, where we could calculate ICC values, we also evaluated the informativeness of the ICC measure ("icc.b" and "icc.m"). One dataset achieved a very low omnibus test $p$ value $(p<0.001)$ while the other two were not significant, possibly due to few replicates available and an extremely sparse signal. In contrast, the biological/technical covariates were less "universal": they were informative only on specific datasets. Overall, the relation to the CpG island ("cpg.loc") was the most informative, followed by the position in the reference gene ("refgene.pos") and probe type ("probe.type"). For chromosome location ("chr") and DNase I hypersensitive site ("dhs"), they were only significant in 4 and 10 datasets, respectively.

A statistically valid covariate needs to be independent of the $p$ values under the null [40]. We verified this condition using stratified histograms as suggested by [40]: the distribution of larger $p$ values had an approximate uniform distribution across the strata of the covariate. Additional file 6: Figure S3 \& S4 gave representative examples for each type of covariate.

\section{Evaluation of the performance of covariate-adaptive FDR control methods on simulated EWAS datasets}

Most of the existing covariate-adaptive FDR methods rely on the independence assumption, i.e., the hypotheses are not correlated. However, for EWAS data, the methylation levels of neighboring $\mathrm{CpGs}$ are usually correlated, violating the independence assumption. It is not clear whether these methods can still control FDR at the target level while retaining the high statistical power for EWAS data. We thus use simulation, where we know the ground truth, to study the performance of the methods. To mimic the correlation structure of EWAS data, we used a real EWAS dataset [50] as a template. We drew random samples from the control group to create two groups of equal sample size and added random or correlated differential signals of varying strength and density ("Methods"). We simulated covariates with varying degrees of informativeness and compared the performance of AdaPT, BL, CAMT, FDRreg, IHW, and the traditional $\mathrm{BH}$ and ST procedure (Table 2).

We first studied the performance under random signal setting, where the DMPs were randomly distributed. We observed that all the methods controlled the false discovery proportion close to or under the target level (5\%) across settings (Fig. 2a). As expected, $\mathrm{BH}$ procedure was more conservative than ST procedure when the signal was dense. IHW was generally the most conservative, especially in the dense signal setting. AdaPT was severely conservative in the sparse signal and less informative covariate setting. CAMT was also conservative in that setting, but to a lesser degree. In terms of statistical power, all the covariate-adaptive methods performed better than or similar to the $\mathrm{BH} / \mathrm{ST}$ procedure when the covariate was informative, indicating their ability to exploit the covariate information (Fig. 2b). CAMT and AdaPT were more powerful than other methods when the signal was dense, or the covariate was highly informative.

However, when the signal was sparse and the covariate was less informative, AdaPT was significantly less powerful than other methods. In contrast, FDRreg and BL performed slightly better than or similar to ST across settings while the performance of IHW varied according to the underlying signal density. IHW was less powerful than FDRreg and BL when the signal was dense, and even slightly less powerful than ST when the covariate was not very informative. As the signal became sparser, IHW was more powerful than FDRreg and BL. We also simulated correlated signals, where the DMPs tended to cluster together. Nevertheless, the pattern remained similar, indicating the robustness of these methods to correlated EWAS signals (Additional file 6: Figure S5). Taken together, all these covariate methods could control the FDR for EWAS data, and their optimal power depended on the specific signal structure as well as the informativeness of the covariate. The favorable performance under the sparse signal makes CAMT and IHW promising candidates for EWAS data since the sparse signal scenarios are statistically more challenging.

\section{Improved detection power of covariate-adaptive FDR control methods on real EWAS datasets}

We next applied IHW, CAMT, AdaPT, FDRreg, and BL to the 61 real EWAS datasets with the 14 aforementioned covariates in addition to the $\mathrm{BH}$ and ST procedure. We first compared the run time of these covariateadaptive methods with different covariates. IHW was almost an order of magnitude faster than the next fastest method (BL) while FDRreg and AdaPT were computationally the most intensive (Additional file 6: Figure S6). The run time of CAMT and AdaPT was sensitive to the number of categories for categorical covariates (e.g., "dhs" and "chr") while other methods were less so. In 
Table 2 Description of the methods evaluated

\begin{tabular}{|c|c|c|c|}
\hline & Method description & R package & Ref \\
\hline $\mathrm{BH}$ & $\begin{array}{l}\text { The classic procedure. } p \text { values for } m \text { hypotheses are ordered from the smallest to the largest. Given a } \\
\text { target FDR level } a \text {, the ith hypothesis is rejected if the } p \text { value is less than the threshold } a \frac{i}{m} \text {. }\end{array}$ & stats (p.adjust) & [28] \\
\hline ST & $\begin{array}{l}\text { The global proportion of null hypotheses is estimated and used to adjust the threshold in the } \mathrm{BH} \\
\text { procedure. Less conservative than } \mathrm{BH} \text { when the signal is not sparse. }\end{array}$ & $\begin{array}{l}\text { qvalue } \\
\text { (qvalue) }\end{array}$ & [29] \\
\hline FDRreg & $\begin{array}{l}\text { Covariates are allowed to influence the prior probability of null. The rejection rule is based on local } \\
\text { false discovery rate (IFDR) under the two-component mixture model. }\end{array}$ & $\begin{array}{l}\text { FDRreg } \\
\text { (FDRreg) }\end{array}$ & [39] \\
\hline IHW & $\begin{array}{l}\text { Tests are divided into groups based on the covariate. Each group is associated with a weight, and the } \\
\text { weight is used to adjust the threshold in the } \mathrm{BH} \text { procedure. Only one covariate is allowed. }\end{array}$ & $\begin{array}{l}\text { ihw } \\
\text { (ihw) }\end{array}$ & [40] \\
\hline$B L$ & $\begin{array}{l}\text { A regression framework is used to estimate the proportion of null hypotheses conditional on observed } \\
\text { covariates. The estimates are used to adjust the threshold in the } \mathrm{BH} \text { procedure. }\end{array}$ & $\begin{array}{l}\text { swfdr } \\
\left(I m \_p i 0\right)\end{array}$ & [42] \\
\hline AdaPT & $\begin{array}{l}\text { Covariates are allowed to influence both the null probability and the } p \text { value distribution under the } \\
\text { alternative. The rejection rule is based on IFDR and an adaptive approach is implemented to control } \\
\text { FDR in finite sample. }\end{array}$ & $\begin{array}{l}\text { adaptMT } \\
\text { (adapt_glm) }\end{array}$ & [43] \\
\hline CAMT & $\begin{array}{l}\text { Covariates are allowed to influence both the null probability and the } p \text { value distribution under the } \\
\text { alternative. A new rejection rule is designed to be robust to model mis-specification. }\end{array}$ & $\begin{array}{l}\text { CAMT } \\
\text { (camt.fdr) }\end{array}$ & [51] \\
\hline
\end{tabular}

terms of detection power, the ST procedure dominated the $\mathrm{BH}$ procedure and never detected less DMPs (Additional file 6: Figure S7). We thus used the ST procedure as the baseline and computed the log fold change in the number of detected DMPs between a method-covariate combination and ST. Figure 3 gave an overview of the detection power for all method-covariate combinations across all datasets, ordered by the estimated signal density. Clearly, none of the methods dominated ST and power loss could occur for all methods when the covariate was uninformative, or the signal density was very low. When the signal density was high (Fig. 3, top), AdaPT and CAMT could significantly improve the detection power with informative covariates and lost little power for less informative ones. BL was slightly more powerful than ST, while FDRreg was in the opposite direction. IHW, on the other hand, was significantly less powerful than ST for most covariates. However, when the signal became less dense (Fig. 3, bottom), CAMT and IHW were overall more powerful than other methods when appropriate covariates were used. In contrast, AdaPT suffered a significant power loss on a number of datasets, regardless of the covariates used. Power loss was also observed for FDRreg; however, to a lesser degree than AdaPT. Interestingly, BL was relatively robust and did not lose power in these situations, but the power improvement was also not extensive (Additional file 6: Figure S8A). Overall, the results on the real data agreed well with those from simulations.

We next ranked the detection power of the covariates on each dataset for each method separately (1-the worst, 12-the best). Among statistical covariates, "mean," "sd.b," "mad," and "precision" outperformed others (Fig. 3, top panel), consistent with the omnibus test results (Fig. 1b). The covariate "mean" was the most informative and ranked top for all methods. Even in datasets such as EWAS33, where there was no obvious correlation between the $p$ value and covariates measuring the variance ("sd.m", "sd.b", "mad"), a stronger correlation was observed for the covariate "mean" (Additional file 6: Figure S9). Although biological/technical covariates' performance was not as universal, they could improve detection power for specific datasets. For example, "cpg.loc" was highly informative for EWAS19 and could improve the detection power for all methods. Therefore, selecting the most relevant covariate(s) is important to achieve optimal performance.

In terms of the detection power with the best covariate, CAMT, followed by IHW, had apparent advantages over other methods (Additional file 6: Figure S8B, S10) with little power loss, indicating its potential to improve the detection power for underpowered studies. Interestingly, the omnibus test $p$ values of the covariates (Fig. 1b) coincided well with the improvement in detection power across methods (Additional file 6: Figure S11), suggesting that the omnibus test could be potentially used to select the most informative covariate before applying those covariate-adaptive methods. Additional file 6: Figure S10 presented the heat map based on the most significant covariate. Besides using the most informative covariate, combining all informative covariates is also possible for methods that accommodate multiple covariates such as CAMT. We tried both strategies using CAMT. We could see that both approaches achieved quite robust results (Additional file 6: Figure S12), the power improvement (top panel) was similar to or slightly better than the best covariate "mean".

\section{Further validation of the detected DMPs by covariate- adaptive FDR methods}

The covariate-adaptive FDR methods improved the detection power over traditional methods using 


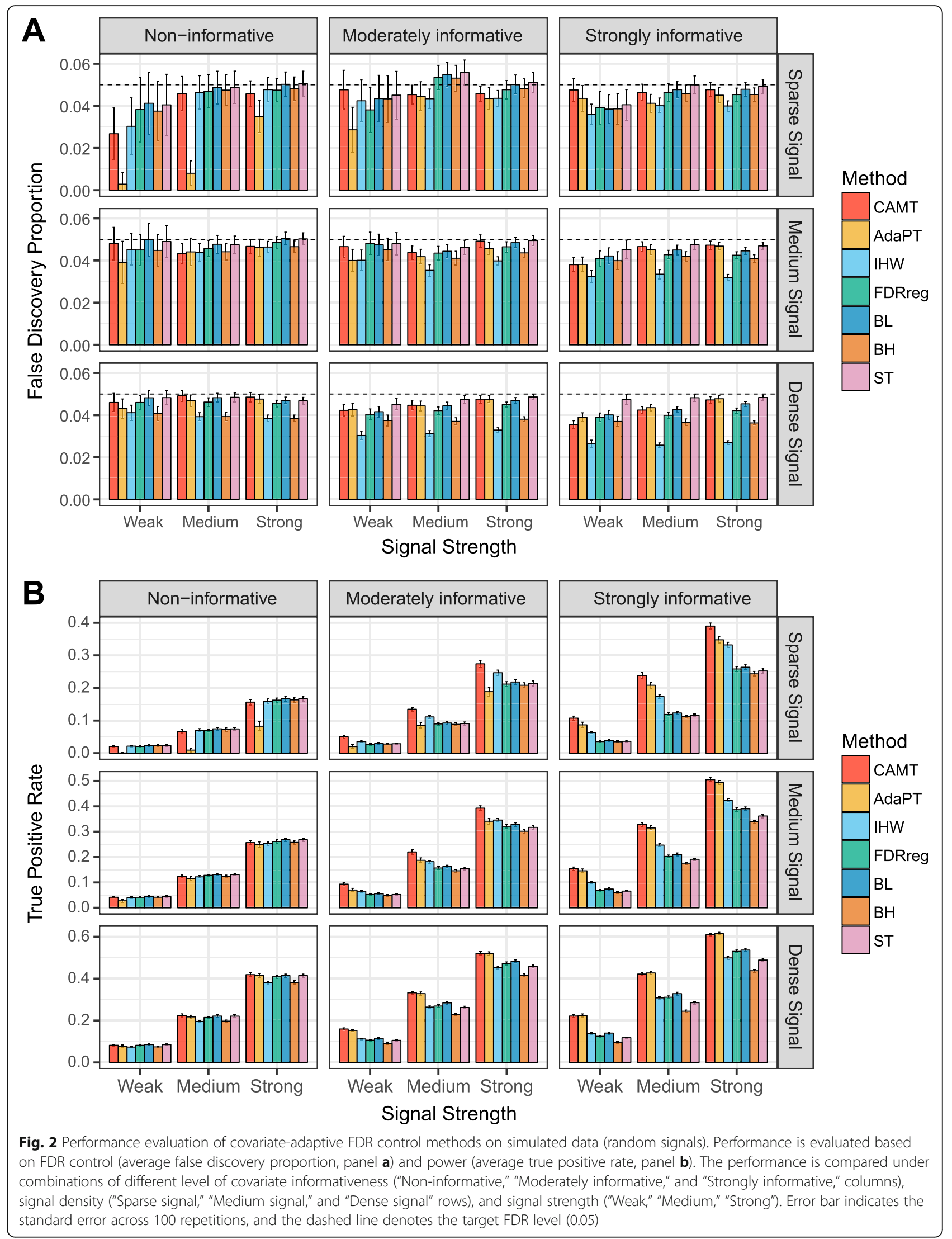




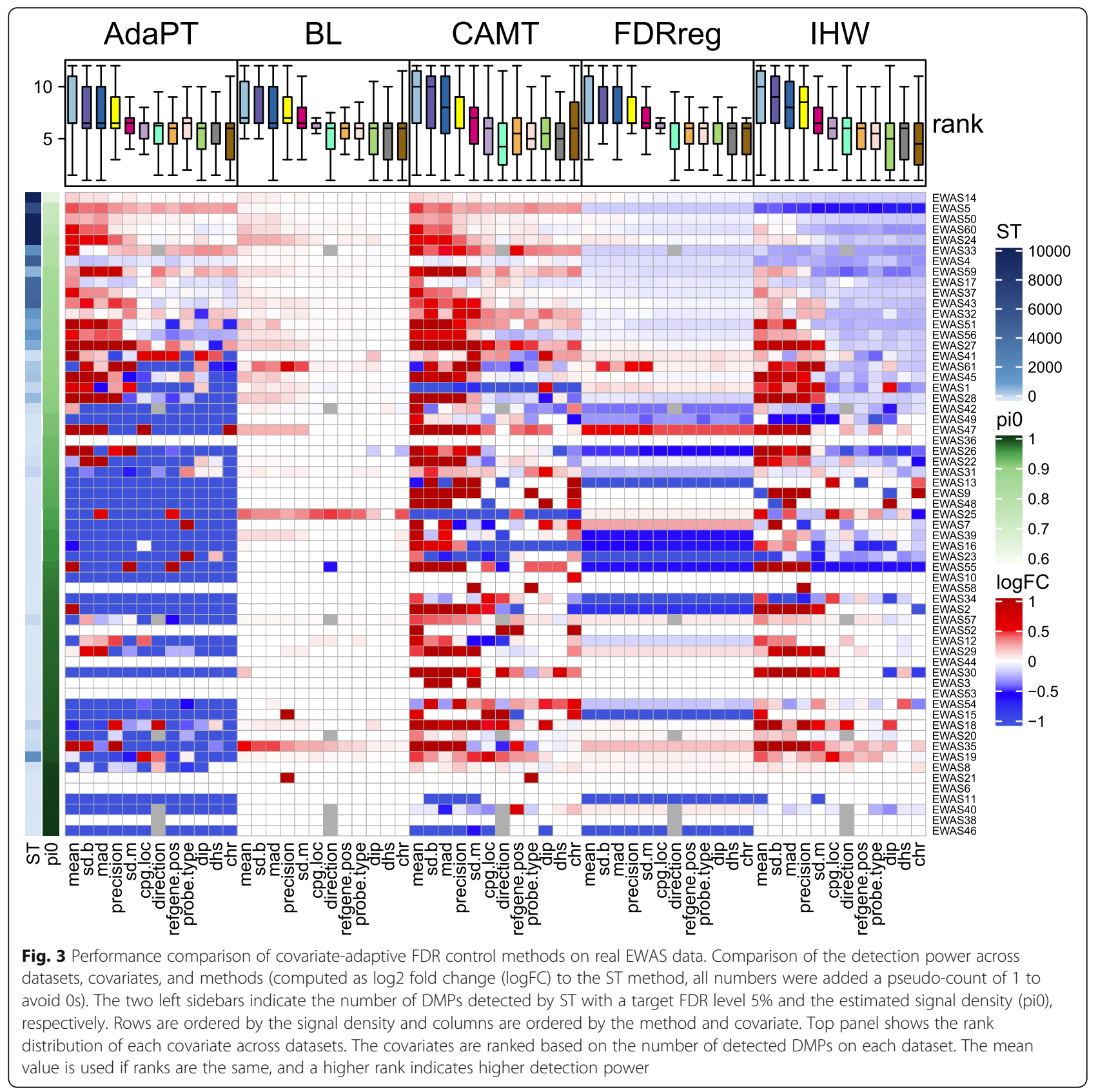

informative covariates, and their results covered the majority of the BH detected DMPs (Additional file 3: Table S3). In this section, we further demonstrated the credibility of the improved detection power from different perspectives using several example datasets. We will focus on CAMT and IHW since they have the overall best performance in the simulation studies.

\section{Down-sampling analysis}

The objective of the down-sampling analysis is to see whether the covariate-adaptive methods have improved power to detect the DMPs based on the full dataset (denoted as "fDMPs") at a smaller sample size. To achieve this end, we down-sampled a large dataset EWAS51 $(n=216$, human fetal alcohol spectrum disorder) [52] and performed CAMT with different covariates on the down-sampled datasets. We first defined a list of "gold standard" fDMPs by applying Bonferroni correction $(\alpha=0.05)$ to the association $p$ values based on the full dataset. We then compared the percentage of these fDMPs detected by CAMT with different covariates to BH/ST at different sample sizes (Fig. 4). None of the procedures could detect any fDMPs when the sample size was too small $(n<30)$. Significant differences in 


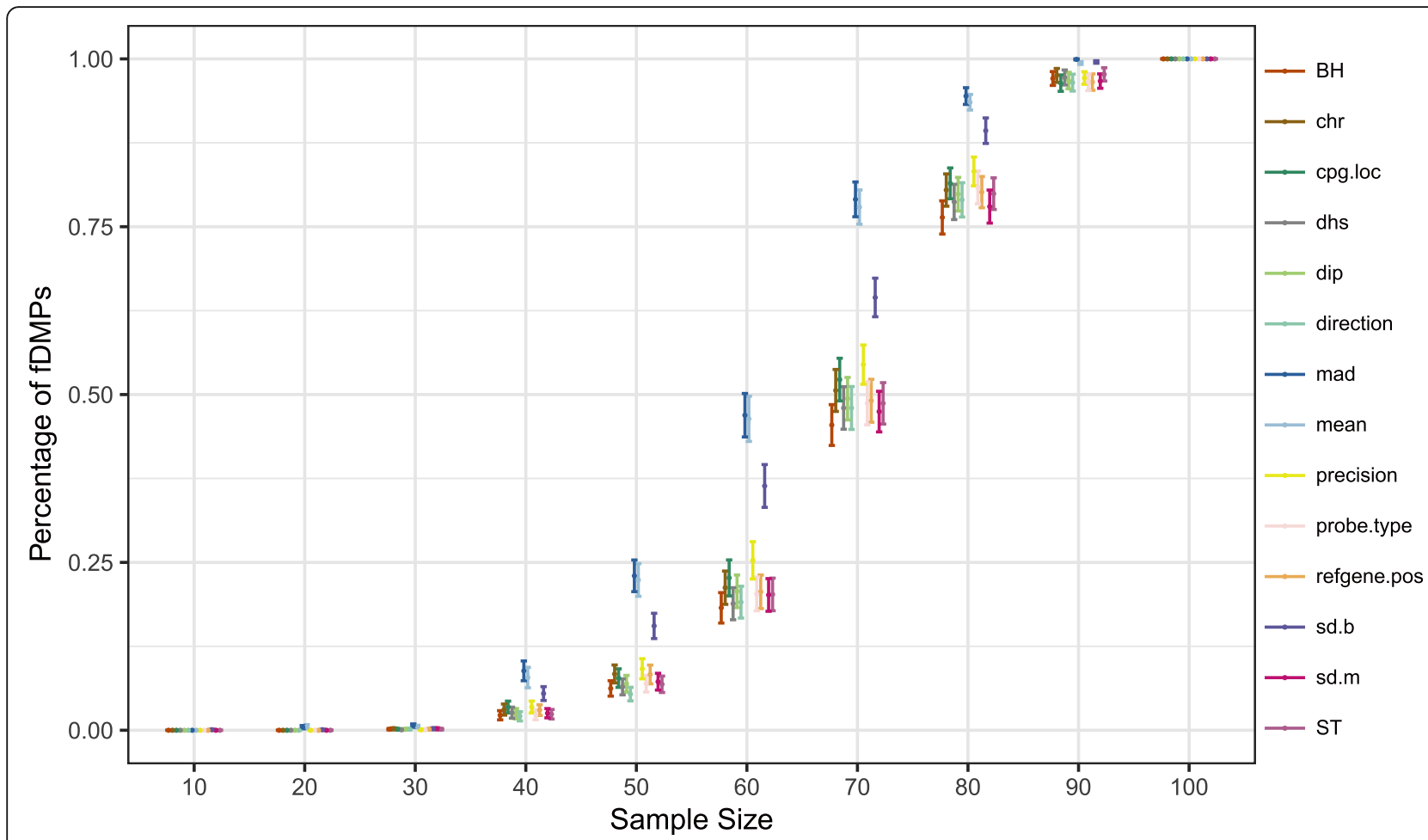

Fig. 4 Covariate-adaptive FDR control methods increase the detection power based on the down-sampling analysis. The results are based on the CAMT method. Y-axis shows the percentage of fDMPs (DMPs using the full dataset) recovered at sample size 10, 20, 30, 40, 50, 60, 70, 80, 90, and 100 for each group. Error bar indicates the standard error

detection power were observed starting from $n=40$, and peaked at $n=70$. As the sample size increased continuously, the power differences became smaller and reached almost $100 \%$ for all methods at $n=100$. Therefore, covariate-adaptive FDR methods could be particularly helpful for a moderately powered study (i.e., power neither too low nor sufficiently high). Notably, covariates "mean," "mad," and "sd.b" outperformed other covariates for this dataset. Similar results can be found for IHW, where we observed a similar trend (Additional file 6: Figure S13).

\section{Detection of age-associated DMPs}

To see whether the covariate-adaptive FDR methods have a better power to detect age-associated DMPs (denoted as "aDMPs"), we defined a list of "gold standard" aDMPs $(n=583)$ derived from two independent studies based on purified blood cell types as described in [46]. We then performed a detailed analysis based on EWAS45, an age EWAS using the peripheral whole blood [25]. For this dataset, all the methods detected more DMPs than ST using informative covariates (Fig. 5). FDR adjustment by the covariate "mean" led to the highest detection power followed by these ICC and variancebased covariates. CAMT, AdaPT, and IHW were overall more powerful than FDRreg and BL with the same informative covariate. We next focused on the list of gold standard aDMPs and compared the distribution of their significance rank among all probes for different methodcovariate combinations. We would expect a much lower rank if a procedure was efficient in detecting aDMPs. Based on IHW, a much lower rank for aDMPs was observed using covariates "mean," "sd.b," and "mad," which are the covariates that also yielded high detection power (Fig. 5a, b). A similar pattern was observed for other methods (Additional file 6: Figure S14). Notably, the covariate "mean" achieved the best rank for all the methods. We thus further compared the rank distribution of aDMPs for the five FDR-adaptive methods using the covariate "mean" (Fig. 5c). IHW, AdaPT, and CAMT achieved a significantly lower median rank than other methods, indicating their improved ability to enrich aDMPs with an informative covariate. We repeated the same analysis on another age EWAS dataset (EWAS27) [53] and arrived a similar conclusion (Additional file 6: Figure S15). Taken together, adaptive FDR control with informative covariates not only increased detection power but also improved the power to retrieve biologically relevant DMPs.

\section{Detection of smoking-associated DMPs}

Smoking has been tightly related to DNA methylation change [54-56]. Here we re-analyzed the dataset from an EWAS of smoking [57] (EWAS20) using IHW and 


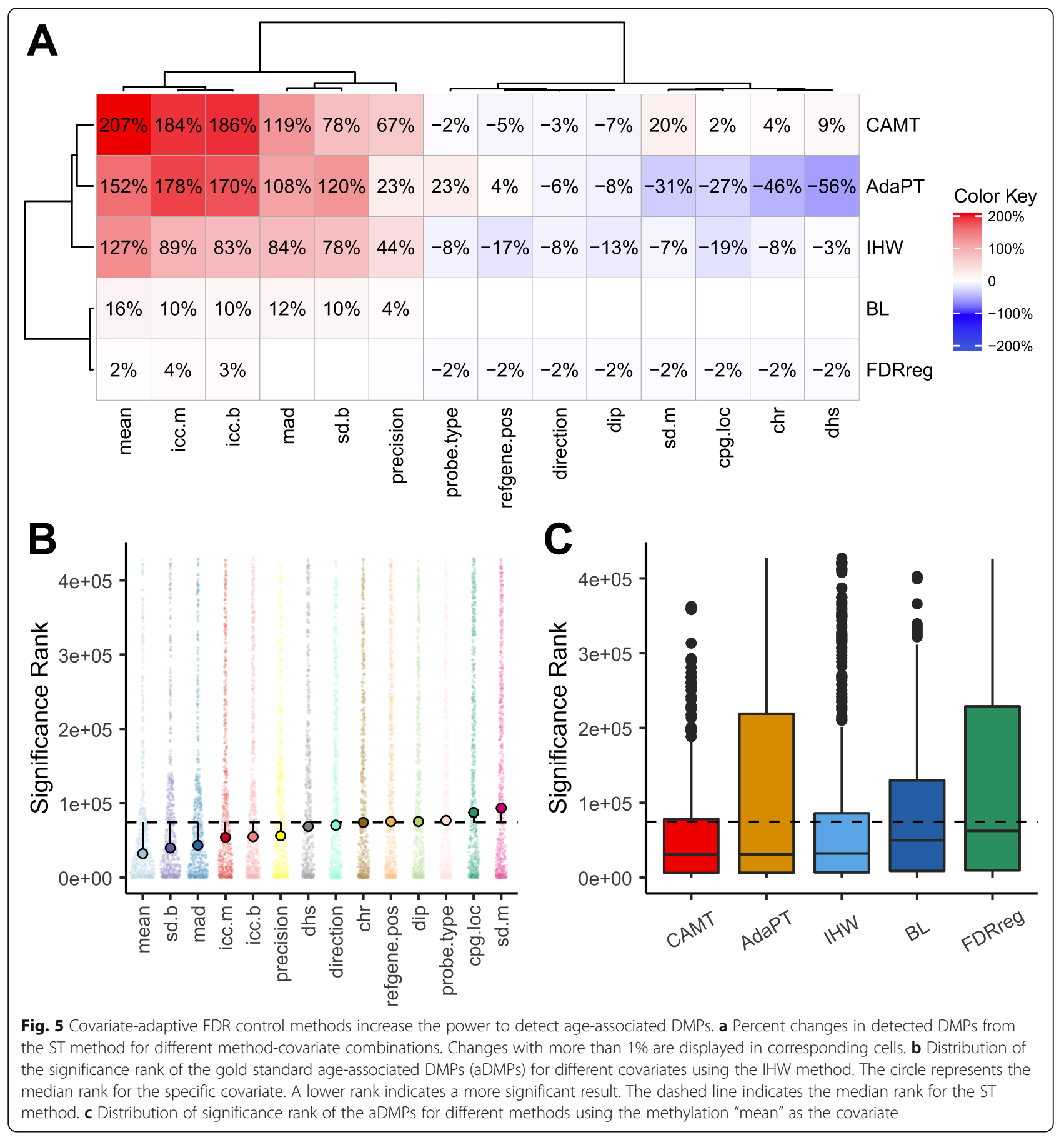

CAMT. The majority of the 30 smoking-associated DMPs (denoted as "sDMPs") reported by [57] were reproduced in our results (Additional file 4: Table S4A). Compared with BH/ST, which produced the same results on this dataset, IHW detected more sDMPs with covariates "sd.b", "mean," "mad," "precision," and "refgene.pos" (Table 3). Some of these new findings were reported in both European and Chinese populations, including cg19827923, cg19254163, cg07986378, cg15159987, and cg22132788 [58].
Two of the sDMPs (cg04885881 and cg22132788) were also among the list of smoking-related CpGs reported at least three times in a systematic review [59].

In addition, cg00300637, cg17924476, and cg24688690 recovered by the covariate "mean" were all located in the body of AHRR, a gene which encodes aryl-hydrocarbon receptor repressor and is known to be associated with smoking [60, 61]. Moreover, we noted that cg18092474, which was discovered by covariates "sd.b," "mean," and 
Table 3 Additional smoking-associated DMPs detected by IHW

\begin{tabular}{|c|c|c|c|c|}
\hline mean & $\operatorname{mad}$ & sd.b & precision & refgene.pos \\
\hline cg04885881 ${ }^{\#}$ & $\mathrm{cg} 04885881^{\#}$ & cg04885881 & cg19827923* & cg06972908 \\
\hline cg12856965 & cg14179389 & cg22740783 & cg19719391 & \\
\hline cg26337070 & cg22740783 & cg19827923* & cg07986378* & \\
\hline cg03147185 & $\operatorname{cg} 26337070$ & cg19719391 & $\operatorname{cg} 14712058$ & \\
\hline cg13185177 & cg19827923* & cg17924476 & $\operatorname{cg} 15159987^{*}$ & \\
\hline cg19719391 & cg19719391 & cg08149865 & & \\
\hline cg00300637 & cg17924476 & cg17476951 & & \\
\hline cg17924476 & $\operatorname{cg} 22132788^{* \#}$ & $\operatorname{cg} 19254163^{*}$ & & \\
\hline cg24688690 & cg08149865 & cg07986378* & & \\
\hline cg037749957 & cg02526790 & cg18092474 & & \\
\hline cg08149865 & cg17476951 & cg06972908 & & \\
\hline $\operatorname{cg} 17476951$ & $\operatorname{cg} 13151811$ & cg08149682 & & \\
\hline cg19254163* & cg07986378* & cg14712058 & & \\
\hline cg07986378* & cg08730245 & cg15159987* & & \\
\hline cg08730245 & cg18092474 & & & \\
\hline $\operatorname{cg} 10322443$ & cg01207684 & & & \\
\hline cg18092474 & cg06972908 & & & \\
\hline cg01207684 & cg25683268 & & & \\
\hline cg16519923 & cg08149682 & & & \\
\hline cg06972908 & cg14712058 & & & \\
\hline cg25683268 & cg15159987* & & & \\
\hline $\operatorname{cg} 15159987^{*}$ & & & & \\
\hline
\end{tabular}

* reported in both European and Chinese population

\# reported at least three times

"mad" at the same time, was located $1500 \mathrm{bp}$ upstream of the transcription start site (TSS) of the CYP1A1 gene, which encodes a member of the cytochrome P450 superfamily of enzymes and was reported to be associated with maternal smoking in newborns [62-64]. Here we show that smoking is also associated with CYP1A1 methylation in adults' peripheral blood. The mean methylation level at cg18092474 for both former and current smokers was significantly lower than never smokers (Additional file 6: Figure S16). Polycyclic aromatic hydrocarbons (PAHs), products of cigarette smoking, were shown to induce the expression of CYP1A1 [65]. Therefore, we speculate that PAHs may upregulate the expression of CYP1A1 by hypomethylation at the cg18092474 position. These results were also supported by CAMT (Additional file 4: Table S4B), which had substantial overlap with the IHW results (Additional file 6: FigureS17).

\section{Potential of using EWAS $p$ value from a related disease/ phenotype as a covariate}

When there are published EWAS data from a similar disease or the same disease but from a different tissue or cell type, we may want to use the published data to improve the detection power of a new EWAS since we expect signal sharing across datasets. Covariate-adaptive FDR methods provide a convenient way to exploit previous data by using the association $p$ value as a covariate. To investigate such possibility, we performed analysis based on EWAS28 and EWAS29, two datasets from EWAS of systemic lupus erythematosus (SLE) using CD19+ B cells and CD4+ T cells, respectively [66]. The signal in EWAS28 (539 DMPs) was much stronger than that of EWAS29 (26 DMPs) according to results from ST. Thus, we used the $z$-score transformed $p$ value from EWAS28 as the covariate to adjust the $p$ value of EWAS29 to see whether we can improve the detection power of a less-powered study. We first verified the assumption of independence under the null using stratified histograms (Additional file 6: Figure S18). Next we applied the omnibus test for assessing the informativeness. The omnibus test showed the new covariate " $p$ value" was highly informative $(p=0.002)$, and it appeared to be more informative than the previously studied covariates for all the covariate-adaptive methods (Fig. 6a). Application of IHW with the new " $p$ value" covariate detected far more DMPs than other covariates (Fig. 6b). Remarkably, it recovered many DMPs detected by EWAS28 but otherwise missed by EWAS29 alone (Fig. 6b). According to Gene Ontology (GO) enrichment analysis based on the genes detected using the new covariate, three out of the top five GO enrichment terms belonged to type I interferon (INF I)-related biological processes (Fig. 6c, all $p$ value $<1 \mathrm{E}-20$ ), which plays an important role in SLE and serves as a therapeutic target [67]. Although these terms were also enriched in the results of the ST method, they were not among the top 10 terms (Additional file 5: Table S5). Similar results can be found by applying CAMT (Additional file 6: Figure S19A\&B). Collectively, the use of $p$ value from a related EWAS as the covariate in FDR control serves as a convenient way to integrate previous knowledge to enrich signal and is a promising approach to an underpowered study.

\section{Discussion}

In this study, we explored the use of start-of-the-art covariate-adaptive FDR control methods in multiple testing correction for epigenome-wide association testing. We also studied the performance of various EWASrelated covariates based on a large collection of EWAS data. We show that all these covariate-adaptive methods could control the false discovery rate at the target level and were substantially more powerful than traditional FDR control methods such as Benjamini-Hochberg's step-up procedure [28] and Storey's $q$ value procedure [29], once informative covariates were used. Compared to the benchmarking simulation study in [44], which simulated the $p$ values directly, we simulated original 


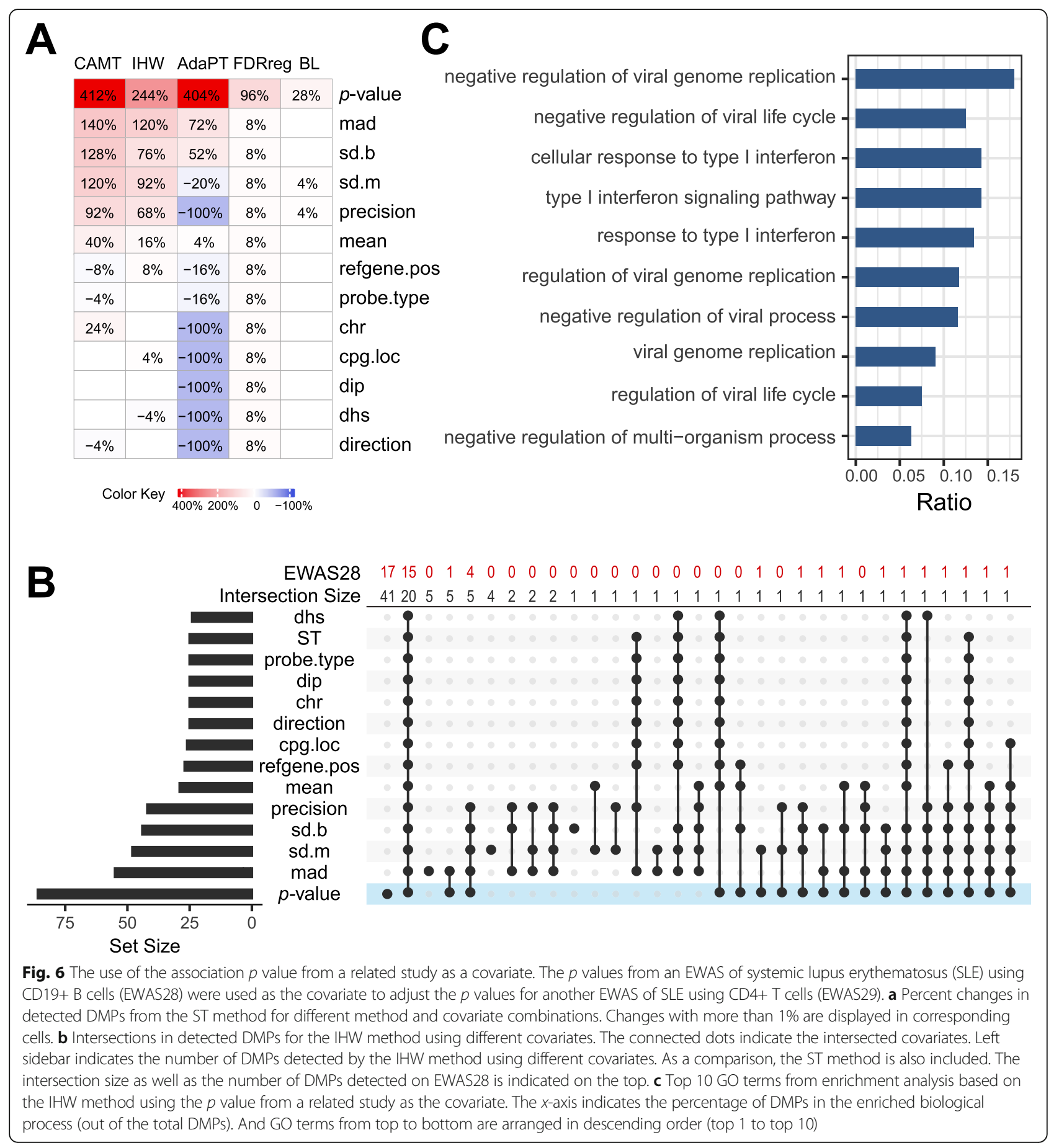

methylation data, upon which $p$ values were calculated. In this way, we preserved the typical correlation structure among $p$ values observed in real EWAS data, and thus the evaluation of false discovery rate control was more rigorous. In addition, we propose an omnibus test for assessing the informativeness of the covariates, investigate the performance of various EWAS-relevant covariates, and benchmark the methods on a large number of real EWAS datasets.
For the 61 real EWAS datasets, the most informative covariate could improve the detection power by a median of $68 \%$ and $25 \%$ for CAMT and IHW, the two best performing methods, compared to the ST procedure. Remarkably, for 9 EWAS datasets, where BH/ST procedure did not make any discovery, CAMT and IHW were able to make positive findings in four and one of the datasets using informative covariates, respectively. We further demonstrated with concrete examples that the 
additional DMPs recovered were biologically meaningful and could help reveal more biological insights. Given the enormous cost associated with epigenome-wide profiling, using covariate-adaptive multiple testing could potentially rescue an underpowered study, reduce the discovery cost, and ultimately shorten the bench to bedside cycle time.

Although the investigated covariate-adaptive FDR control methods all maintained the target FDR level, their power varied tremendously depending on the covariate informativeness and signal density. CAMT and AdaPT were most powerful when the signal density was high or the covariate was strongly informative, suggesting their strong ability to utilizing the underlying information. However, when the signal became sparser and the covariate less informative, AdaPT suffered from a great power loss, which was consistent with the observation in [45]. Although some remedy could be possibly invoked to compensate the power loss as suggested in [45], no implementation has been available in the AdaPT package as of January 2020. On the other hand, CAMT was less susceptible to such power degradation, although some power loss was still observed for less informative covariates in real data. On the contrary, IHW had the best performance when the signal was sparse, but the performance deteriorated with increasing signal density. In circumstances where the signal is dense, and the covariate is not very informative, IHW could be less-powerful than ST. The power of FDRreg and $\mathrm{BL}$, on the other hand, were overall more similar to ST. Significant power gain was only observed when the signal was relatively dense, and the covariate was very informative. In the real data, $\mathrm{BL}$ was more robust than FDRreg. Although there was no uniformly most powerful procedure, CAMT and IHW stood out due to their excellent performance under the sparse signal setting, where power improvement is most needed. Also, IHW was an order-of-magnitude faster than other methods, making it particularly appealing for large-scale analyses.

All the covariate-adaptive FDR control methods could lose power if the covariate was not informative. Thus, assessment of the informativeness of the covariate was recommended before applying a covariate-adaptive method. The traditional way of assessment relied on statistical graphics such as scatter plots or stratified $p$ value histograms [40]. Such an assessment was not effective when the signal was sparse. We thus developed a powerful omnibus test to test the dependence between the $p$ value and the covariate accounting for potential nonlinearity and signal sparsity. We show that the omnibus test $p$ value correlated well with the power improvement for various covariate-adaptive methods. However, the optimal omnibus $p$ value cutoff to achieve significant power gain differed by method and simulation studies may help determine the best cutoff.

The validity of these covariate-adaptive methods depends on the assumption that the $p$ value is independent of the covariate under the null. This assumption is usually assessed empirically by stratified histograms: for large $p$ values (e.g., $p$ values $\geq 0.5$ ), the distribution should appear uniform across strata. A variant of the omnibus test could be used to formally assess the assumption of independence under the null. Specifically, the omnibus test can be focused on the large $p$ values $(p$ value $>0.5$, considered to be dominantly null $p$ values) and tailor the $p$ value cutoff points to be more equally spaced (e.g., $p^{(i)} \in\{0.1,0.2,0.4,0.6,0.8,0.9\}$-quantile of the $p$ values). We show by additional simulations (Additional file 6: Figure S20, simulation details in the legend) that this test is very powerful and can detect a very moderate deviation from the assumption. Therefore, in practice, we could apply both types of omnibus test simultaneously and select covariates that are significant in the original test (i.e., overall dependency between $p$ value and covariate) and not significant in the suggested variant (i.e., the dependency is not due to violation of the independence assumption under the null).

For EWAS data, we investigated 14 potential useful covariates. We found that statistical covariates are more informative than biological/technical covariates, as the former are more directly related to statistical power. In addition, statistical covariates could also be informative of the prior null probability. For example, the variance of the feature is considered to be a good indicator of the null probability [40] since an invariant feature is less likely to be differential. Variance-based filters have been frequently used to exclude invariant genomic features to reduce multiple testing burden [68] and increase statistical power. One advantage of using covariate-adaptive methods is that filtering is no longer necessary, and the method could automatically down-weight these less likely features according to their variances. Interestingly, "sd.b" performed better than "sd.m" on the real data even though the association tests were performed on the M-values. This could be explained by the fact that "sd.b" as the standard deviation on the Beta-value might be a more biologically relevant measurement of methylation variability and is more informative of the null probability. The strong performance of the covariate "mean" is somewhat unexpected and may be explained by two reasons, which are related to the prior null probability and statistical power, respectively. First, DMPs are enriched in certain regions such as the promoters [30,31], where the methylation levels are similar-either low or high (Additional file 6: Figure S3A). Second, the statistical power to detect DMPs in the middle of the methylation 
spectrum is higher than those in the two ends, where the methylation changes are constrained (Additional file 6: Figure S3B). The covariate "dip" was the least informative among all statistical covariates since the majority of CpGs, including those DMPs, have unimodal distributions and the dip statistic is not very informative. Compared to statistical covariates, external covariates were less universal than statistical covariates, and they were informative only for specific datasets. For instance, the covariate "cpg.loc" was very powerful for EWAS19 since DMPs in pancreatic ductal adenocarcinoma were enriched in CpG islands [20].

Besides the traditional covariates, we also explored the use of $p$ value from the same or similar disease/ phenotype as a covariate, and we demonstrated a substantial power gain. Using $p$ value as a covariate provides a convenient way to conduct integrative analysis without the need for sophisticated statistical modeling or access to the raw data. Other possible covariates, which are not explored here, include the detection $p$ value, existence of SNP in the probe, possible crosshybridization [69] and probe-level reproducibility [70] from an independent study. With large-scale genome annotation endeavors such as the ENCODE project [71], an increasing number of genomic annotations are now available and they can all be used as potential covariates. The proposed framework can be used to evaluate their relevance for EWAS.

Finally, as several covariates may be informative for a particular dataset, finding the optimal combination of them will be an interesting topic. Except for IHW, all the covariate-adaptive methods can accommodate multiple covariates. Coupling the omnibus test and these methods may be a feasible solution.

\section{Conclusions}

Covariate-adaptive FDR control methods can significantly increase the detection power for EWAS using informative covariates. The choice of the optimal method depends on the underlying signal density and the informativeness of the covariate. The informativeness of the covariate can be assessed based on the proposed omnibus test. For a dense signal, CAMT and AdaPT are the most powerful; for a sparse signal, CAMT and IHW are most promising.

\section{Methods}

Data

We collected 58 methylation datasets from GEO and split them into 61 EWAS datasets according to the phenotype, tissue source, and cell type under the following criteria: (1) the platform is Infinium Human Methylation 450K BeadChip; (2) sample size is no less than 100 . Note that the majority of the 61 EWAS datasets are for different outcomes with some for the same outcome such as smoking. For datasets with raw IDAT files (the raw intensity data from the beadchip) available, $\mathrm{R}$ function minfi::read.metharray.exp (version 1.26.2) [72] was used to extract Beta-values and detection $p$ values. Otherwise, we obtained the data from the soft file on GEO via GEOquery::getGEO (version 2.48.0) [73]. The annotation file of Infinium Human Methylation $450 \mathrm{~K}$ BeadChip was obtained from $\mathrm{R}$ package IlluminaHumanMethylation450kanno.ilmn12.hg19

(version 0.6.0) [74].

\section{Quality control}

The normalization of raw data was carried out by preprocessQuantile [75] function from minfi package. Samples with mean detection $p$ value greater than or equal to 0.01 were removed, and probes with median detection $p$ value across all samples less than or equal to 0.01 were filtered. Probes designed for sex chromosomes or autosomal probes co-hybridizing to sex chromosomes (crossreactive) [69] were also filtered. Besides, probes with common SNPs either at the CpG interrogation site or at the single nucleotide extension site were excluded, regardless of minor allele frequency.

\section{Differential methylation analysis and multiple testing correction}

Before performing multiple testing correction, surrogate variable analysis was conducted using $\mathrm{R}$ package isva (version 1.9) [76] and SmartSVA (version 0.1.3) [46] to capture significant sources of methylation variability such as cellular heterogeneity, age, and other unknown batch effects. The resulting surrogate variables were treated as covariates in the linear regression model to address potential confounding effects of cellular heterogeneity and batch effects. Differential methylation analysis was performed using the function cpg.assoc from $\mathrm{R}$ package CpGassoc (version 2.60) [77] and conducted on M-values [78] (logit.transform = TRUE). The association $p$ values were then corrected for multiple testing by various FDR control methods. Traditional FDR control methods Benjamini-Hochberg's step-up procedure $(\mathrm{BH})$ and Storey's $q$ value procedure (ST) were performed using the R package stats (version 3.5.3) [79] and qualue (version 2.12.0) [80], respectively. Covariate-adaptive FDR control methods IHW, CAMT, BL, AdaPT, and FDRreg were performed using $\mathrm{R}$ package $I H W$ (version 1.8.0) [40], CAMT (version 1.0) [44], swfdr (version 1.6.0) [42], adaptMT (version 1.0.0) [43], and FDRreg (version 0.1) [39] with default settings (except FDRreg, theoretical null type was used to achieve more robust performance). See details in Table 2. A target FDR level of $5 \%$ was used throughout the study. 


\section{An omnibus test for assessing the informativeness of the covariates}

The informativeness of the covariate is assessed by testing the association between the $p$ value from differential methylation analysis and the covariate. Due to potential sparsity and nonlinearity of the association signal, rankbased tests (e.g., Spearman's rank test and KruskalWallis test) may not be powerful since the vast majority of the $p$ values are not expected to be associated with the covariate under the null. We thus develop an omnibus test, which zooms into the low $p$ value region and addresses nonlinearity by categorizing the covariate if it is continuous. Denote $p_{1}, p_{2}, \ldots, p_{m}$ the $p$ values for $m$ CpGs and $x_{1}, x_{2}, \ldots, x_{m}$ the values for covariate $X$. For a given $p$ value cutoff $p^{(i)}, i=1, \ldots, I$, we convert the $p$ value into a binary variable $P^{(i)}\left(\leq p^{(i)},>p^{(i)}\right)$. We next consider two scenarios:

(1) $X$ is categorical. To quantify the association between $P^{(i)}$ and $X$, we use the $X^{2}$ test and denote $q^{(i)}$ as the $-\log p$ value from the $\chi^{2}$ test. We then define the omnibus test statistic for a categorical covariate as

$$
t^{o}=\max _{i} q^{(i)}
$$

which pools information across different dichotomizations.

(2) $X$ is continuous. We further categorize $X$ to address potential nonlinear effects. For a given number of categories $n^{(j)}, j=1, \ldots, J$, we convert $X$ into a categorical variable $X^{(j)}$ with equal category size. To quantify the association between $P^{(i)}$ and $X^{(j)}$, we use both $\chi^{2}$ test and Cochran-Armitage test for trend [81] and denote $q_{1}^{(i, j)}$ and $q_{2}^{(i, j)}$ as the $-\log p$ value from the respective tests. The use of Cochran-Armitage test for trend is to compensate potential power loss of $\chi^{2}$ test when $P^{(i)}$ and $X^{(j)}$ have a monotone relationship. We then define the omnibus test statistic for a continuous covariate as

$$
t^{o}=\max _{i, j}\left\{\max \left[q_{1}^{(i, j)}, q_{2}^{(i, j)}\right]\right\}
$$

We next use permutation to assess the significance. Denote the test statistic under permutation as $t_{k}^{p}(k=1$, $\ldots, K)$. The omnibus test $p$ value is calculated

$$
\text { pval }=\frac{1+\sum_{k} \mathrm{I}\left(t_{k}^{p} \geq t^{o}\right)}{1+K} .
$$

For both simulated and real datasets, we use $p^{(i)} \in$ $\{0.001,0.005,0.01,0.05,0.1,0.2\}$-quantile of the $p$ values $, n^{(j)} \in\{2,4,8,16,32\}, K=999$. Since nearby CpGs are usually correlated, to satisfy the exchangeability assumption of the permutation test, we ordered CpGs by their genomic position and down-sampled them to achieve near zero auto-correlation ( $\mathrm{R}$ stats::acf) in real data analysis.

\section{Simulation studies \\ Simulations for comparing covariate-adaptive FDR control methods}

To preserve the correlation structure observed in real data, we based our simulation on a real EWAS dataset [50]. We drew random samples from the control group to create two groups of equal sample size $\left(n_{1}=n_{2}=80\right)$, based on which we added differential signals. For demonstration purpose, we only used CpGs from the chromosome $13(m=11,808)$. To study the impact of signal density, signal strength, and informativeness of the covariate, we simulated $27(3 \times 3 \times 3)$ settings with a varying degree of signal density (sparse, medium, and dense), signal strength (weak, moderate, and strong), and covariate informativeness (none, moderate, and strong). We first simulated "random signals," where the differential CpGs were randomly distributed along the chromosome. Specifically, we first generated the covariate $x_{i} \sim N(0,1), i=1, \ldots, m$. Given $x_{i}$, we generated $\pi_{0 i}$, the probability of being the null for the $i$ th hypothesis, as

$$
\pi_{0 i}=\frac{\exp \left(\eta_{0}+c x_{i}\right)}{1+\exp \left(\eta_{0}+c x_{i}\right)}
$$

where we set $\eta_{0} \in\{3.5,2.5,1.5\}$, representing sparse, medium, and dense signals $(3 \%, 8 \%$, and $18 \%$ under no covariate effect), and $c \in\{0,1,1.5\}$, representing a noninformative, moderately informative, and strongly informative covariate, respectively. With $\pi_{0 i}$, we generated the differential status $H_{i} \sim \operatorname{Bernoulli}\left(1-\pi_{0 i}\right)$. If a CpG was differential, i.e., $H_{i}=1$, we added a methylation difference $f_{i} \in\{0.27,0.33,0.45\}$ (on $\mathrm{M}$-value) to samples from one group, representing a weak, moderate, and strong effect. Linear regression was then performed to generate the association $p$ values, which were further analyzed by various covariate-adaptive FDR procedures, along with the covariate. We also simulated "correlated signals," where the differential CpGs were clustered on the chromosome. To achieve this end, we generated $x_{i}$ from a autoregressive model ( $\mathrm{R}$ stats::arima.sim, ar $=0.75$ ).

\section{Simulations for studying the performance of the omnibus test}

We simulated $m=10,000$ hypotheses and investigated both categorical and continuous covariates. For a categorical covariate, we randomly sampled $x_{i} \in\{1,2,3,4,5\}, i=1, \ldots, m$. For a continuous covariate, we generated $x_{i} \sim N(0,1), i=1$, ..., $m$. Next, we used the same aforementioned simulation strategy on standardized $x_{i}$ (mean 0 , sd 1$)$ to generate $H_{i}$, 
where $\eta_{0} \in\{3.5,2.5,1.5\}$, representing sparse, medium, and dense signals, and $c \in\{0,0.25,0.375\}$, representing no dependence, weak dependence, and moderate dependence on the covariate, respectively. To simulate a nonlinear dependency for a continuous covariate, we replaced $x_{i}$ with $x_{i}^{2}$ in generating $\pi_{0 i}$. Next, we generated the $z$-score $z_{i} \sim N(0,1)$, if $H_{i}=0$, and $z_{i} \sim N(2.68,1)$ if $H_{i}=1$. We finally generated the $p$ value based on $1-\Phi\left(z_{i}\right)$, where $\Phi$ is the c.d.f of the standard normal.

\section{Covariates}

We investigated 14 covariates "mean," "sd.b," "sd.m," "mad," "dip," "precision," "direction," "icc.b," “icc.m," "refgene.pos," "cpg.loc," "chr," "dhs," and "probe.type." The definition, calculation, and representation of these covariates could be found in Table 1 . If the covariate was categorical, we coded it as a factor in $\mathrm{R}$ and transformed into a model matrix if needed. If the covariate was continuous, we applied natural cubic spline transformation to allow a certain degree of nonlinearity. We used Bayesian information criterion (BIC) to determine the optimal degree of freedom on several real datasets, and a degree of freedom of 6 was found to be adequate to achieve satisfactory results. We thus set the degree of freedom to be 6 in the natural spline $(n s(\mathrm{x}, \mathrm{df}=6)$ in $\mathrm{R}$ package splines, version 3.5.0) [79]. Besides, the same covariates were used for both pi0 and $\mathrm{f} 1$ if needed (CAMT and AdaPT).

\section{Down-sampling analysis}

We used EWAS51 (112 human fetal alcohol spectrum disorder samples with 104 controls) to perform downsampling analysis. CpGs with Bonferroni-corrected $p$ value below 0.05 based on the full sample size were defined as fDMPs. Since EWAS51 was of binary phenotype, we subsampled each phenotype to a sample size $10,20,30,40,50,60,70,80,90$, and 100 . At each sample size, 100 replications were performed.

\section{GO and KEGG enrichment analysis}

GO and KEGG enrichment analysis was done by gometh function of R package missMethyl (version 1.16.0) [82]. All CpG labels in the array data were used as background.

\section{Visualization}

For data cleaning and transformation, $\mathrm{R}$ package tidyverse (version 1.2.1) [83] was applied. Figures were generated and arranged by $\mathrm{R}$ package ggplot2 (version 3.1.1) [84], ggforce (version 0.3.0) [85], ComplexHeatmap (version 2.1.0) [86], UpSetR (version 1.4.0) [87], ggpubr (version 0.1.7) [88], and cowplot (version 1.0.0) [89]. Clustering heatmaps were generated with distance measure "euclidean" and clustering method "ward.D".

\section{Supplementary information}

Supplementary information accompanies this paper at (https://doi.org/10. 1186/s13059-020-02001-7).

Additional file 1: Table S1. Description of the Datasets.

Additional file 2: Table S2. Informativeness statistics for the 14 covariates based on the omnibus test.

Additional file 3: Table S3. Percentage of BH-significant DMPs recovered by IHW with different covariates.

Additional file 4: Table S4A. Reproducing the smoking EWAS results on EWAS20. Table S4B. Additional smoking-associated DMPs detected by CAMT.

Additional file 5: Table S5. Top 20 GO Terms based on ST Results. Additional file 6: Supplementary figures.

Additional file 7: Review history.

\section{Abbreviations}

EWAS: Epigenome-wide association studies; FDR: False discovery rate; FWER: Family-wise error rate; $\mathrm{BH}$ : Benjamini-Hochberg step-up procedure; ST: Storey's q value procedure; DMPs: Differentially methylated CpG positions: LFDR: The conditional local FDR; FDRreg: FDR regression; IHW: Independent hypothesis weighting; ASH: Adaptive shrinkage; BL: Boca and Leek's FDR regression; AdaPT: Adaptive $p$ value thresholding; CAMT: Covariate Adaptive Multiple Testing; GEO: Gene Expression Omnibus; ICC: Intraclass correlation coefficient; fDMPs: Differentially methylated CpG positions based on the full dataset; aDMPs: Age-associated differentially methylated CpG positions; sDMPs: Smoking-associated differentially methylated CpG positions; TSS: Transcription start site; PAHs: Polycyclic aromatic hydrocarbons; SLE: Systemic lupus erythematosus; INF I: Type I interferon; GO: Gene Ontology; ENCODE: The Encyclopedia of DNA Elements

\section{Acknowledgements}

We want to thank Yongshuai Jiang form Harbin Medicine University for providing a tidy candidate list of EWAS datasets.

\section{Review history}

The review history is available as Additional file 7.

\section{Peer review information}

Anahita Bishop was the primary editor on this article and managed its editorial process and peer review in collaboration with the rest of the editorial team.

\section{Authors' contributions}

JC conceived, designed, and supervised the study with $\mathrm{JH}$ and $\mathrm{XZ}$. JH coordinated and oversaw the bioinformatics data analysis. JC developed the test and performed the simulation studies with the help from YY. LB, LW, ZA, and SR downloaded and cleaned the public data. $L B, B C$, and LW wrote the $R$ workflow scripts for 450k methylation data differential analysis. LB performed all the analysis on real EWAS datasets. $L B, J H$ and JC are the major contributors in writing the manuscript. All authors read, revised, and approved the final manuscript.

\section{Funding}

$\mathrm{LB}, \mathrm{BC}$, and $\mathrm{J}$ were supported by the National Key Research and Development Plan of China Grants (No. 2018YFA0107802), the National Natural Science Foundation of China (NSFC) General Program (No. 81570122, 81770205), the National Key Research and Development Program (No. 2016YFC0902800), Innovative research team of high-level local universities in Shanghai, and the Shanghai Municipal Education Commission-Gaofeng Clinical Medicine Grant Support (No. 20161303), Samuel Waxman Cancer Research Foundation and Center for HPC at Shanghai Jiao Tong University. XZ was supported by the US National Science Foundation grants (DMS1830392, DMS-1811747). JC was supported by Mayo Clinic Center for Individualized Medicine.

\section{Availability of data and materials}

All the EWAS datasets analyzed during the current study are publicly available on the Gene Expression Omnibus (GEO, http://www.ncbi.nlm.nih. 
gov/geo) [90] at corresponding GEO accession provided in Additional file 1 : Table S1. The data and source code to reproduce all the figures in this study are available on GitHub repository at the site [91] and Zenodo [92].

\section{Ethics approval and consent to participate}

All the results in this study are based on publicly available data and do not require ethics approval.

\section{Consent for publication}

Not applicable.

\section{Competing interests}

The authors declare that they have no competing interests.

\section{Author details}

'State Key Laboratory of Medical Genomics, Shanghai Institute of Hematology, National Research Center for Translational Medicine, Rui-Jin Hospital, Shanghai Jiao Tong University School of Medicine, Shanghai Jiao Tong University, 197 Ruijin Er Road, Shanghai 200025, China. ²Department of General Surgery, Rui-Jin Hospital, Shanghai Jiao Tong University, 197 Ruijin Er Road, Shanghai 200025, China. ${ }^{3}$ Division of Digital Health Sciences, Mayo Clinic, 200 1st St SW, Rochester, MN 55905, USA. ${ }^{4}$ Department of Statistics, Texas A\&M University, Blocker 449D, College Station, TX 77843, USA. ${ }^{5}$ Division of Biomedical Statistics and Informatics, Department of Health Sciences Research and Center for Individualized Medicine, Mayo Clinic, 200 1st St SW, Rochester, MN 55905, USA

\section{Received: 8 October 2019 Accepted: 17 March 2020}

\section{Published online: 06 April 2020}

\section{References}

1. You JS, Kelly TK, De Carvalho DD, Taberlay PC, Liang G, Jones PA. OCT4 establishes and maintains nucleosome-depleted regions that provide additional layers of epigenetic regulation of its target genes. Proc Natl Acad Sci U S A. 2011:108:14497-502.

2. Bocker MT, Hellwig I, Breiling A, Eckstein V, Ho AD, Lyko F. Genome-wide promoter DNA methylation dynamics of human hematopoietic progenitor cells during differentiation and aging. Blood. 2011;117:e182.

3. Calvanese V, Fernández AF, Urdinguio RG, Suárez-Alvarez B, Mangas C, Pérez-García V, Bueno C, Montes R, Ramos-Mejía V, Martínez-Camblor P, et al. A promoter DNA demethylation landscape of human hematopoietic differentiation. Nucleic Acids Res. 2012;40:116-31.

4. Smith ZD, Meissner A. DNA methylation: roles in mammalian development. Nat Rev Genet. 2013;14:204-20.

5. Augui $S$, Nora EP, Heard E. Regulation of X-chromosome inactivation by the X-inactivation Centre. Nat Rev Genet. 2011;12:429-42.

6. Ben-Avraham D, Muzumdar RH, Atzmon G. Epigenetic genome-wide association methylation in aging and longevity. Epigenomics. 2012:4:503-9.

7. Ciccarone F, Tagliatesta S, Caiafa P, Zampieri M. DNA methylation dynamics in aging: how far are we from understanding the mechanisms? Mech Ageing Dev. 2018;174:3-17.

8. Morgan AE, Davies TJ, Mc Auley MT. The role of DNA methylation in ageing and cancer. Proc Nutr Soc. 2018;77:412-22.

9. Meier K, Recillas-Targa F. New insights on the role of DNA methylation from a global view. Front Biosci (Landmark Ed). 2017:22:644-68.

10. Baylin SB, Jones PA. Epigenetic determinants of cancer. Cold Spring Harb Perspect Biol. 2016;8:a019505.

11. Fernandez-Sanles A, Sayols-Baixeras S, Subirana I, Degano IR, Elosua R. Association between DNA methylation and coronary heart disease or other atherosclerotic events: a systematic review. Atherosclerosis. 2017;263:325-33.

12. De Jager PL, Srivastava G, Lunnon K, Burgess J, Schalkwyk LC, Yu L, Eaton ML, Keenan BT, Ernst J, McCabe C, et al. Alzheimer's disease: early alterations in brain DNA methylation at ANK1, BIN1, RHBDF2 and other loci. Nat Neurosci. 2014:17:1156-63.

13. Lunnon K, Smith R, Hannon E, De Jager PL, Srivastava G, Volta M, Troakes C, Al-Sarraj S, Burrage J, Macdonald R, et al. Methylomic profiling implicates cortical deregulation of ANK1 in Alzheimer's disease. Nat Neurosci. 2014;17: 1164-70.

14. Cashen AF, Schiller GJ, O'Donnell MR, DiPersio JF. Multicenter, phase II study of decitabine for the first-line treatment of older patients with acute myeloid leukemia. J Clin Oncol. 2010;28:556-61.
15. Estey EH. Epigenetics in clinical practice: the examples of azacitidine and decitabine in myelodysplasia and acute myeloid leukemia. Leukemia. 2013; 27:1803-12

16. Santini V, Allione B, Zini G, Gioia D, Lunghi M, Poloni A, Cilloni D, Sanna A Masiera E, Ceccarelli M, et al. A phase II, multicentre trial of decitabine in higher-risk chronic myelomonocytic leukemia. Leukemia. 2018;32:413-8.

17. Rivera CM, Ren B. Mapping human epigenomes. Cell. 2013;155:39-55.

18. Horvath S, Ritz BR. Increased epigenetic age and granulocyte counts in the blood of Parkinson's disease patients. Aging (Albany NY). 2015;7:1130-42.

19. Yang IV, Pedersen BS, Liu A, O'Connor GT, Teach SJ, Kattan M, Misiak RT, Gruchalla R, Steinbach SF, Szefler SJ, et al. DNA methylation and childhood asthma in the inner city. J Allergy Clin Immunol. 2015;136:69-80.

20. Nones K, Waddell N, Song S, Patch AM, Miller D, Johns A, Wu J, Kassahn KS, Wood D, Bailey P, et al. Genome-wide DNA methylation patterns in pancreatic ductal adenocarcinoma reveal epigenetic deregulation of SLITROBO, ITGA2 and MET signaling. Int J Cancer. 2014;135:1110-8.

21. Guo X, Chen X, Wang J, Liu Z, Gaile D, Wu H, Yu G, Mao G, Yang Z, Di Z, et al. Multi-generational impacts of arsenic exposure on genome-wide DNA methylation and the implications for arsenic-induced skin lesions. Environ Int. 2018;119:250-63.

22. Liu D, Zhao L, Wang Z, Zhou X, Fan X, Li Y, Xu J, Hu S, Niu M, Song X, et al. EWASdb: epigenome-wide association study database. Nucleic Acids Res. 2019:47:D989-93.

23. Zhao L, Liu D, Xu J, Wang Z, Chen Y, Lei C, Li Y, Liu G, Jiang Y. The framework for population epigenetic study. Brief Bioinform. 2018;19:89-100.

24. Bonferroni CE. Teoria statistica delle classi e calcolo delle probabilità. Pubblicazioni del R Istituto Superiore di Scienze Economiche e Commerciali di Firenze. 1936:8:3-62.

25. Tan Q, Heijmans BT, Hjelmborg JV, Soerensen M, Christensen K, Christiansen L. Epigenetic drift in the aging genome: a ten-year follow-up in an elderly twin cohort. Int J Epidemiol. 2016;45:1146-58.

26. Nordlund J, Backlin CL, Wahlberg P, Busche S, Berglund EC, Eloranta ML, Flaegstad T, Forestier E, Frost BM, Harila-Saari A, et al. Genome-wide signatures of differential DNA methylation in pediatric acute lymphoblastic leukemia. Genome Biol. 2013;14:r105.

27. Butcher DT, Cytrynbaum C, Turinsky AL, Siu MT, Inbar-Feigenberg M, Mendoza-Londono R, Chitayat D. CHARGE and kabuki syndromes: genespecific DNA methylation signatures identify epigenetic mechanisms linking these clinically overlapping conditions. Am J Hum Genet. 2017;100:773-88.

28. Benjamini $Y$, Hochberg $Y$. Controlling the false discovery rate: a practical and powerful approach to multiple testing. J R Stat Soc Ser B. 1995;57:289300.

29. JD S. A direct approach to false discovery rates. J R Stat Soc Ser B. 2002;64: 479-98.

30. Liu Y, Duong W, Krawczyk C, Bretschneider N, Borbely G, Varshney M, Zinser C, Schar P, Ruegg J. Oestrogen receptor beta regulates epigenetic patterns at specific genomic loci through interaction with thymine DNA glycosylase. Epigenetics Chromatin. 2016;9:7.

31. Declerck K, Remy S, Wohlfahrt-Veje C, Main KM, Van Camp G, Schoeters G, Vanden Berghe W, Andersen HR. Interaction between prenatal pesticide exposure and a common polymorphism in the PON1 gene on DNA methylation in genes associated with cardio-metabolic disease risk-an exploratory study. Clin Epigenetics. 2017:9:35.

32. Zhang Y, Fukui N, Yahata M, Katsuragawa Y, Tashiro T, Ikegawa S, Lee MT. Identification of DNA methylation changes associated with disease progression in subchondral bone with site-matched cartilage in knee osteoarthritis. Sci Rep. 2016:6:34460.

33. Li Y, Chen JA, Sears RL, Gao F, Klein ED, Karydas A, Geschwind MD, Rosen $\mathrm{HJ}$, Boxer AL, Guo W, et al. An epigenetic signature in peripheral blood associated with the haplotype on 17q21.31, a risk factor for neurodegenerative tauopathy. PLoS Genet. 2014;10:e1004211.

34. Karlsson L, Barbaro M, Ewing E, Gomez-Cabrero D, Lajic S. Epigenetic alterations associated with early prenatal dexamethasone treatment. Endocr Soc. 2019:3:250-63.

35. Chen J, Just AC, Schwartz J, Hou L, Jafari N, Sun Z, Kocher JP, Baccarelli A, Lin X. CpGFilter: model-based CpG probe filtering with replicates for epigenome-wide association studies. Bioinformatics. 2016;32:469-71.

36. Bose M, Wu C, Pankow JS, Demerath EW, Bressler J, Fornage M, Grove ML, Mosley TH, Hicks C, North K, et al. Evaluation of microarray-based DNA methylation measurement using technical replicates: the atherosclerosis risk in communities (ARIC) study. BMC Bioinformatics. 2014;15:312. 
37. Roeder K, Wasserman L. Genome-wide significance levels and weighted hypothesis testing. Stat Sci. 2009;24:398-413.

38. Cai TT, Sun W. Simultaneous testing of grouped hypotheses: finding needles in multiple haystacks. J Am Stat Assoc. 2009;104:1467-81.

39. Scott JG, Kelly RC, Smith MA, Zhou P, Kass RE. False discovery rate regression: an application to neural synchrony detection in primary visual cortex. J Am Stat Assoc. 2015;110:459-71.

40. Ignatiadis N, Klaus B, Zaugg JB, Huber W. Data-driven hypothesis weighting increases detection power in genome-scale multiple testing. Nat Methods. 2016:13:577-80

41. Stephens M. False discovery rates: a new deal. Biostatistics. 2017;18:275-94.

42. Boca SM, Leek JT. A direct approach to estimating false discovery rates conditional on covariates. PeerJ. 2018;6:e6035.

43. W LLF. AdaPT: an interactive procedure for multiple testing with side information. J R Stat Soc Ser B. 2018:80:649-79.

44. Zhang $X$, Chen J. Covariate adaptive false discovery rate control with applications to Omics-wide multiple testing. arXiv e-prints. arXiv. 2019:1909. 04811. Available from: https://ui.adsabs.harvard.edu/abs/2019arXiv190904811 Z. R package available at https://github.com/jchen1981/CAMT. Accessed 17 Sept 2019.

45. Korthauer K, Kimes PK, Duvallet C, Reyes A, Subramanian A, Teng M, Shukla C, Alm EJ, Hicks SC. A practical guide to methods controlling false discoveries in computational biology. Genome Biol. 2019;20:118.

46. Chen J, Behnam E, Huang J, Moffatt MF, Schaid DJ, Liang L, Lin X. Fast and robust adjustment of cell mixtures in epigenome-wide association studies with SmartSVA. BMC Genomics. 2017;18:413.

47. McGregor K, Bernatsky S, Colmegna I, Hudson M, Pastinen T, Labbe A, Greenwood CM. An evaluation of methods correcting for cell-type heterogeneity in DNA methylation studies. Genome Biol. 2016;17:84.

48. Leek JT, Scharpf RB, Bravo HC, Simcha D, Langmead B, Johnson WE, Geman D, Baggerly K, Irizarry RA. Tackling the widespread and critical impact of batch effects in high-throughput data. Nat Rev Genet. 2010;11:733-9.

49. Wang X, Laird PW, Hinoue T, Groshen S, Siegmund KD. Non-specific filtering of beta-distributed data. BMC Bioinformatics. 2014;15:199.

50. Wijnands KP, Chen J, Liang L, Verbiest MM, Lin X, Helbing WA, Gittenberger-de Groot AC, van der Spek PJ, Uitterlinden AG, Steegers-Theunissen RP. Genomewide methylation analysis identifies novel $\mathrm{CpG}$ loci for perimembranous ventricular septal defects in human. Epigenomics. 2017;9:241-51.

51. Zhang X, Chen J. Covariate Adaptive False Discovery Rate Control with Applications to Omics-Wide Multiple Testing. arXiv preprint arXiv:1909. 04811. 2019.

52. Portales-Casamar E, Lussier AA, Jones MJ, Maclsaac JL, Edgar RD, Mah SM, Barhdadi A, Provost S, Lemieux-Perreault LP, Cynader MS, et al. DNA methylation signature of human fetal alcohol spectrum disorder. Epigenetics Chromatin. 2016;9:25.

53. Tserel L, Kolde R, Limbach M, Tretyakov K, Kasela S, Kisand K, Saare M, Vilo J, Metspalu A, Milani L, Peterson P. Age-related profiling of DNA methylation in CD8+ T cells reveals changes in immune response and transcriptional regulator genes. Sci Rep. 2015;5:13107.

54. Lee KW, Pausova Z. Cigarette smoking and DNA methylation. Front Genet. 2013;4:132.

55. Prince C, Hammerton G, Taylor AE, Anderson EL, Timpson NJ, Davey Smith $\mathrm{G}$, Munafo MR, Relton $\mathrm{CL}$, Richmond RC. Investigating the impact of cigarette smoking behaviours on DNA methylation patterns in adolescence. Hum Mol Genet. 2019;28:155-65.

56. Joehanes R, Just AC, Marioni RE, Pilling LC, Reynolds LM, Mandaviya PR, Guan W, Xu T, Elks CE, Aslibekyan S, et al. Epigenetic signatures of cigarette smoking. Circ Cardiovasc Genet. 2016;9:436-47.

57. Tsaprouni LG, Yang TP, Bell J, Dick KJ, Kanoni S, Nisbet J, Vinuela A, Grundberg E, Nelson CP, Meduri E, et al. Cigarette smoking reduces DNA methylation levels at multiple genomic loci but the effect is partially reversible upon cessation. Epigenetics. 2014;9:1382-96.

58. Zhu X, Li J, Deng S, Yu K, Liu X, Deng Q, Sun H, Zhang X, He M, Guo H, et al. Genome-wide analysis of DNA methylation and cigarette smoking in a Chinese population. Environ Health Perspect. 2016;124:966-73.

59. Gao X, Jia M, Zhang Y, Breitling LP, Brenner H. DNA methylation changes of whole blood cells in response to active smoking exposure in adults: a systematic review of DNA methylation studies. Clin Epigenetics. 2015;7:113.

60. Bojesen SE, Timpson N, Relton C, Davey Smith G, Nordestgaard BG. AHRR (cg05575921) hypomethylation marks smoking behaviour, morbidity and mortality. Thorax. 2017;72:646-53.
61. Philibert RA, Beach SR, Brody GH. Demethylation of the aryl hydrocarbon receptor repressor as a biomarker for nascent smokers. Epigenetics. 2012;7: $1331-8$.

62. Joubert BR, Haberg SE, Nilsen RM, Wang X, Vollset SE, Murphy SK, Huang Z, Hoyo C, Midttun O, Cupul-Uicab LA, et al. 450K epigenome-wide scan identifies differential DNA methylation in newborns related to maternal smoking during pregnancy. Environ Health Perspect. 2012;120:1425-31.

63. Markunas CA, Xu Z, Harlid S, Wade PA, Lie RT, Taylor JA, Wilcox AJ. Identification of DNA methylation changes in newborns related to maternal smoking during pregnancy. Environ Health Perspect. 2014;122:1147-53.

64. Janssen BG, Gyselaers W, Byun HM, Roels HA, Cuypers A, Baccarelli AA, Nawrot TS. Placental mitochondrial DNA and CYP1A1 gene methylation as molecular signatures for tobacco smoke exposure in pregnant women and the relevance for birth weight. J Transl Med. 2017;15:5.

65. Wohak LE, Krais AM, Kucab JE, Stertmann J, Ovrebo S, Seidel A, Phillips DH, Arlt VM. Carcinogenic polycyclic aromatic hydrocarbons induce CYP1A1 in human cells via a p53-dependent mechanism. Arch Toxicol. 2016;90:291-304.

66. Absher DM, Li X, Waite LL, Gibson A, Roberts K, Edberg J, Chatham WW, Kimberly RP. Genome-wide DNA methylation analysis of systemic lupus erythematosus reveals persistent hypomethylation of interferon genes and compositional changes to CD4+ T-cell populations. PLoS Genet. 2013;9: e1003678.

67. Lauwerys BR, Ducreux J, Houssiau FA. Type I interferon blockade in systemic lupus erythematosus: where do we stand? Rheumatology (Oxford). 2014;53: 1369-76.

68. Hackstadt AJ, Hess AM. Filtering for increased power for microarray data analysis. BMC Bioinformatics. 2009;10:11.

69. Chen YA, Lemire M, Choufani S, Butcher DT, Grafodatskaya D, Zanke BW, Gallinger S, Hudson TJ, Weksberg R. Discovery of cross-reactive probes and polymorphic CpGs in the Illumina Infinium HumanMethylation450 microarray. Epigenetics. 2013;8:203-9.

70. Forest M, O'Donnell KJ, Voisin G, Gaudreau H, Maclsaac JL, McEwen LM, Silveira PP, Steiner M, Kobor MS, Meaney MJ, Greenwood CMT. Agreement in DNA methylation levels from the lllumina 450K array across batches, tissues, and time. Epigenetics. 2018;13:19-32.

71. Consortium EP. An integrated encyclopedia of DNA elements in the human genome. Nature. 2012;489:57-74.

72. Aryee MJ, Jaffe AE, Corrada-Bravo H, Ladd-Acosta C, Feinberg AP, Hansen $K D$, Irizarry RA. Minfi: a flexible and comprehensive Bioconductor package for the analysis of Infinium DNA methylation microarrays. Bioinformatics (Oxford, England). 2014;30:1363-9.

73. Davis S, Meltzer PS. GEOquery: a bridge between the gene expression omnibus (GEO) and BioConductor. Bioinformatics. 2007;14:1846-7.

74. Hansen KD. IlluminaHumanMethylation450kanno.IImn12.hg19: Annotation for Illumina's 450k methylation arrays. 2016. R package version 0.6.0. https:/ bioconductor.org/packages/3.5/data/annotation/html/ IlluminaHumanMethylation450kanno.lmn12.hg19.html. Accessed 19 Sept 2019.

75. Touleimat N, Tost J. Complete pipeline for Infinium((R)) Human Methylation 450K BeadChip data processing using subset quantile normalization for accurate DNA methylation estimation. Epigenomics. 2012;4:325-41.

76. Teschendorff AE. isva: Independent Surrogate Variable Analysis. 2017. R package version 1.9. https://CRAN.R-project.org/package=isva. Accessed 17 Sept 2019.

77. Barfield R, Conneely K, Kilaru V. CpGassoc: Association Between Methylation and a Phenotype of Interest. 2017. R package version 2.60. https://CRAN.Rproject.org/package=CpGassoc. Accessed 17 Sept 2019.

78. Du P, Zhang X, Huang CC, Jafari N, Kibbe WA, Hou L, Lin SM. Comparison of Beta-value and M-value methods for quantifying methylation levels by microarray analysis. BMC Bioinformatics. 2010;11:587.

79. Team RC. R: a language and environment for statistical computing. 2018.

80. Storey JD, Bass AJ, Dabney A, Robinson D. qvalue: Q-value estimation for false discovery rate control. 2015. R package version 2.12.0. http://github. com/jdstorey/qvalue. Accessed 17 Sept 2019.

81. Sasieni PD. From genotypes to genes: doubling the sample size. Biometrics. 1997;53:1253-61.

82. Phipson B, Maksimovic J, Oshlack A. miss Methyl: an R package for analyzing data from Illumina's HumanMethylation450 platform. Bioinformatics. 2016; 32:286-8.

83. Wickham H. tidyverse: Easily Install and Load the 'Tidyverse'. 2017. R package version 1.2.1. https://CRAN.R-project.org/package=tidyverse. Accessed 16 Sept 2019. 
84. Wickham H. ggplot2: Elegant Graphics for Data Analysis. New York: Springer-Verlag; 2016.

85. Pedersen TL. ggforce: Accelerating 'ggplot2'. 2019. R package version 0.3.0. https://CRAN.R-project.org/package=ggforce. Accessed 17 Sept 2019.

86. Gu Z, Eils R, Schlesner M. Complex heatmaps reveal patterns and correlations in multidimensional genomic data. Bioinformatics. 2016;32: 2847-9.

87. Gehlenborg N. UpSetR: a more scalable alternative to Venn and Euler diagrams for visualizing intersecting sets. 2017. R package version 1.3.3. https://CRAN.R-project.org/package=UpSetR. Accessed 17 Sept 2019.

88. Kassambara A. ggpubr: 'ggplot2' based publication ready plots. 2018. R package version 0.1.7. https://CRAN.R-project.org/package=ggpubr. Accessed 17 Sept 2019.

89. Wilke CO. cowplot: streamlined plot theme and plot annotations for 'ggplot2'. 2019. R package version 1.0.0. https://CRAN.R-project.org/ package $=$ cowplot. Accessed 17 Sept 2019

90. Barrett T, Wilhite SE, Ledoux P, Evangelista C, Kim IF, Tomashevsky M, Marshall KA, Phillippy KH, Sherman PM, Holko M, et al. NCBI GEO: archive for functional genomics data sets--update. Nucleic Acids Res. 2013;41:D991-5.

91. Huang J, Bai L, Cui B, Wu L, Wang L, An Z, Ruan S, Yue Y, Zhang X, Chen J. Leveraging biological and statistical covariates improves the detection power in epigenome-wide association testing. GitHub. 2019; https:/github. com/JhuangLab/EWASpaper.

92. Huang J, Bai L, Cui B, Wu L, Wang L, An Z, Ruan S, Yue Y, Zhang X, Chen J. Leveraging biological and statistical covariates improves the detection power in epigenome-wide association testing. Zenodo. https://doi.org/10. 5281/zenodo.3692126

\section{Publisher's Note}

Springer Nature remains neutral with regard to jurisdictional claims in published maps and institutional affiliations.

Ready to submit your research? Choose BMC and benefit from:

- fast, convenient online submission

- thorough peer review by experienced researchers in your field

- rapid publication on acceptance

- support for research data, including large and complex data types

- gold Open Access which fosters wider collaboration and increased citations

- maximum visibility for your research: over $100 \mathrm{M}$ website views per year

At $\mathrm{BMC}$, research is always in progress.

Learn more biomedcentral.com/submissions 